This is a self-archived - parallel published version of this article in the publication archive of the University of Vaasa. It might differ from the original.

\title{
Pattern Classification and PSO Optimal Weights Based Sky Images Cloud Motion Speed Calculation Method for Solar PV Power Forecasting
}

Author(s): Zhen, Zhao; Pang, Shuaijie; Wang, Fei; Li, Kangping; Li, Zhigang; Ren, Hui; Shafie-khah, Miadreza

Title: $\quad$ Pattern Classification and PSO Optimal Weights Based Sky Images Cloud Motion Speed Calculation Method for Solar PV Power Forecasting

Year: $\quad 2019$

Version: Accepted manuscript

Copyright (C) 2019 IEEE. Personal use of this material is permitted. Permission from IEEE must be obtained for all other uses, in any current or future media, including reprinting/republishing this material for advertising or promotional purposes, creating new collective works, for resale or redistribution to servers or lists, or reuse of any copyrighted component of this work in other works.

\section{Please cite the original version:}

Zhen, Z., Pang, S., Wang, F., Li, K., Li, Z., Ren, H. \& Shafie-khah, M. (2019). Pattern Classification and PSO Optimal Weights Based Sky Images Cloud Motion Speed Calculation Method for Solar PV Power Forecasting. IEEE Transactions on Industry Applications 55(4), 3331-3342. https://doi.org/10.1109/TIA.2019.2904927 


\title{
Pattern Classification and PSO Optimal Weights Based Sky Images Cloud Motion Speed Calculation Method for Solar PV Power Forecasting
}

\author{
Zhao Zhen, Shuaijie Pang, Fei Wang, Senior Member, IEEE, Kangping Li, Student Member, IEEE, Zhigang Li, \\ Member, IEEE, Hui Ren, Miadreza Shafie-khah, Senior Member, IEEE and João P. S. Catalão, Senior Member, IEEE
}

\begin{abstract}
The motion of cloud over PV power station will directly cause the change of solar irradiance, which indirectly affects the prediction of minute-level PV power. Therefore, the calculation of cloud motion speed is very crucial for $P V$ power forecasting. However, due to the influence of complex cloud motion process, it is very difficult to achieve accurate result using a single traditional algorithm. In order to improve the computation accuracy, a pattern classification and PSO optimal weights-based sky images cloud motion speed calculation method for solar PV power forecasting (PCPOW) is proposed. The method consists of two parts. Firstly, we use k-means clustering method and texture features based on Gray-Level Co-occurrence Matrix (GLCM) to classify the clouds. Secondly, for different cloud classes, we build the corresponding combined calculation model to obtain cloud motion speed. Real data recorded at Yunnan Electric Power Research Institute is used for simulation, the results show that the cloud classification and optimal combination model are effective, and the PCPOW can improve the accuracy of displacement calculation.
\end{abstract}

Index Terms--Cloud Motion Speed; Combined Modeling; Optimal Weights; Pattern Classification; Sky Image, Power Forecasting.

Z. Zhen, S. Pang, F. Wang, K. Li and H. Ren and is with the Department of Electrical Engineering, North China Electric Power University, Baoding 071003, China; F. Wang also with the State Key Laboratory of Alternate Electrical Power System with Renewable Energy Sources (North China Electric Power University), Beijing 102206, China, and also with the Hebei Key Laboratory of Distributed Energy Storage and Microgrid (North China Electric Power University), Baoding 071003, China (e-mail: feiwang@ncepu.edu.cn).

$\mathrm{Z}$. Li is with South China University of Technology, Guangzhou 510006, China (e-mail: lizg16@scut.edu.cn).

M. Shafie-khah is with University of Vaasa, Finland (e-mail: miadreza@gmail.com)

J. P. S. Catalão is with INESC TEC and the Faculty of Engineering of the University of Porto, Porto 4200-465, Portugal, also with CMAST, University of Beira Interior, Covilhã 6201-001, Portugal, and also with INESC-ID, Instituto Superior Técnico, University of Lisbon, Lisbon 1049-001, Portugal (e-mail: catalao@ubi.pt).

\section{INTRODUCTION}

As a significant fashion to utilize the solar energy, the photovoltaic (PV) power has gained burgeoning expansion of late years owing to its merits of no fuel consumption, no pollutant emission, and flexible configuration. However, PV belongs to intermittent power supplies, and to be more exact, there are randomness and volatility in PV output as it is affected by meteorological factors, i.e. solar irradiance, ambient temperature, moisture, wind velocity and barometric pressure, etc. [1]-[3]. These shortcomings may bring rigorous challenges to power balance, security stability and economic operation of the power system [4]-[7]. From the above, we can see that in order to provide a credible understructure for power system scheduling decisionmaking behavior and ameliorate its capacity to consume intermittent power, it is the time to put forward an accurate and effective prediction scheme of PV [8]-[11]. Previously, many researches [12]-[14] do not consider the cloud motion speed in PV power forecasting. The time scale of these prediction methods is 15 minutes, which not only reduces the accuracy sharply under cloudy conditions, but also fails to meet the requirements of real-time grid dispatching. The cloud motion, such as birth, dissipation and deformation, are pivotal elements of the transformation of solar irradiance, thus giving rise to the change of PV output [15]-[16]. Therefore, the investigation of cloud motion has turned out to be one of the extremely critical tasks to complete the above-mentioned forecast methodology.

In the related research of cloud motion, most investigators take advantage of satellite images for analysis and processing [17]-[-20] in an early phase. However, satellite images are not ideal for the elaboration of regional or low cloud info as a result of low spatial and temporal resolution [21]. Therefore, their prediction accuracy cannot meet people's needs. Currently, in order to track the movement of the local cloud (especially over the PV power station) more accurately, scholars obtain the speed of cloud by means of ground-based images.

There are three main ways to calculate the cloud speed: Block-Matching, Optical Flow and feature matching algorithm. The Block-Matching algorithm performs the work by measuring the similarity of the sub blocks between adjacent images. And it is adopted by Chow et al. in [22], Huang in [23] and Peng et al. in [24]. The Optical Flow algorithm is a pixel-level non-rigid 
registration method, according to the assumption that the gray level of the image remains unchanged. Following original approaches presented in Horn and Schunck (HS) in [25] as well as Lucas and Kanade (LK) in [26], Optical Flow algorithm is used by Wood-Bradley in [27], Chow in [28], and Brox in [29]. As for the feature matching algorithm, this is a broad concept. You need to find corresponding features (e.g. Harris points, SIFT points or SURF points) in adjacent images to complete the task of regional matching. Based on the idea of feature matching, Cheng adopted SIFT algorithm in [30], and F Su used SURF algorithm in [31]. Compared with previous studies based on satellite images, these literatures have better prediction accuracy and local applicability. However, a full-fledged and effective cloud tracing method has not been proposed up to present. As a rigid registration method, Block-Matching cannot obtain the non-rigid motion parameters of cloud, such as rotation and deformation. The accuracy of the optical flow, which is based on the assumption that the image grayscale is not changed, is easily disturbed by noise. For example, in the case of uneven illumination, the computational accuracy is on the low side. As for feature matching, because the definition of a feature point usually requires a lot of texture information, it is poorly matched in regions where the texture information is not rich enough. In short, the above three methods have poor robustness. Because the clouds in the sky images may have various forms, there are above demerits that cannot be overcome when we use the same single method for all kinds of clouds. Furthermore, any simple combined calculation modeling without cloud classification is difficult to achieve good results as well.

In this paper, we propose a pattern classification and PSO optimal weights-based sky images cloud motion speed calculation method for solar PV power forecasting. The related works are mainly divided into the following two parts: Firstly, we classify cloud according to the texture feature information of sky images captured by ground-based sky imager. Secondly, for different cloud classes obtained in the previous step, we utilize PSO to optimize the weights of three methods: Block-Matching, Optical Flow and SURF feature matching algorithm, and build the corresponding combined calculation modeling.

In the light of the method proposed, we can choose different calculation strategies (set different weights of three methods in the combined modeling) according to different clouds. In other words, it could improve the weaknesses of the traditional single method in the applicable scope and it is a more universal modeling suitable for most cloud scenes. As for the second chapter, we will give a detailed description of the proposed method "PCPOW". In the third chapter, we will utilize measured data to validate the proposed method and compare the results with those of single common algorithm. Finally, in the fourth chapter, we will summarize the work of the full paper and discuss the future work.

\section{THE METHOD OF PCPOW}

The flowchart of the PCPOW is shown in Fig. 1.

\section{A. Pattern Classification of Clouds}

For clouds in sky images, we can describe them in terms of brightness, size, shape, spectrum, and texture features, etc. As a regional feature, texture is a description of the spatial distribution of each pixel in an image.

We can simply understand that texture consists of texture primitives that are repeated in accordance with certain rules or statistical rules. Because texture can adequately reflect image information, compared with other image features, it can better take into account both the macro nature and the fine structure of images. In this paper, we use the texture features based on GrayLevel Co-occurrence Matrix (GLCM) to classify the clouds.

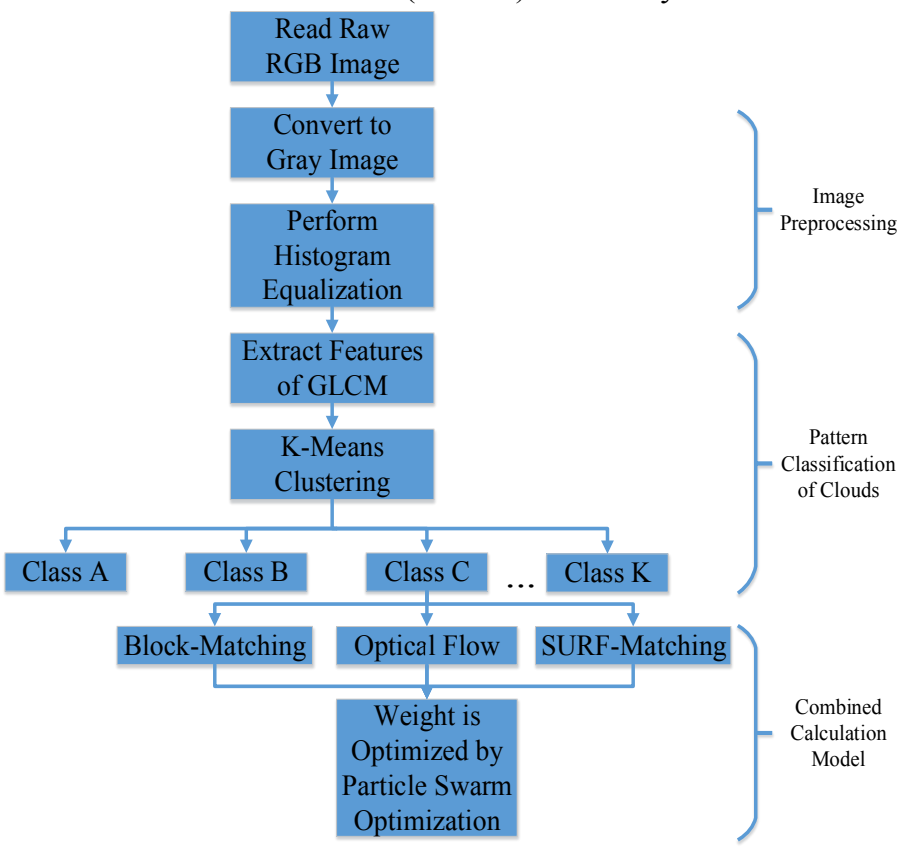

Fig.1 Flowchart of the PCPOW

\section{1) Histogram Equalization}

Many of the sky image textures are not rich enough, and in order to improve the speed of the program, we have compressed the resolution of original sky images. These will lead to the loss of image information, so we must enhance the image firstly.In this paper, we employ histogram equalization to enhance the image.

If the pixels of an image share many gray levels and are evenly distributed, then such images tend to have high contrast and variable gray tones. Histogram equalization is a transformation that can automatically achieve this effect by relying only on the histogram information of the input image.

Its basic idea is to widen the gray levels which contain more pixels in the image, and to compress the gray levels which contain fewer pixels in the image. Thus, the dynamic range of the pixel gray value is extended, and the contrast and the change 
degree of the gray tone hue are improved, and a clearer image is generated.

\section{2) Gray-Level Co-occurrence Matrix}

Take points $(\mathrm{x}, \mathrm{y})$ and $(\mathrm{x}+\mathrm{a}, \mathrm{y}+\mathrm{b})$ as a point pair in the image. The gray value of the point pair is $(i, j)$, and that is to say, the gray value of the point $(\mathrm{x}, \mathrm{y})$ is $\mathrm{i}$, and the gray value of the point $(\mathrm{x}+\mathrm{a}, \mathrm{y}+\mathrm{b})$ is $\mathrm{j}$. Fix $a$ and $b$, and move the point $(\mathrm{x}, \mathrm{y})$ on the whole image, you'll get all sorts of $(i, j)$. Let the gray level of the image be $L$, then the combination of $i$ and $j$ has a total of $L^{2}$ species. In the whole image, the number of occurrences of $(i, j)$ is counted, and then they are normalized to the probability $P_{i j}$, then the square $\left[P_{i j}\right]_{L * L}$ is the GLCM.

For slowly changing textures (i.e. coarse textures), the GLCM has a larger diagonal value, and the two sides are smaller, that is, GLCM tends to be diagonal distributions; on the contrary, for fast changing textures (i.e. fine textures), it tends to be evenly distributed. Obviously, different $(\mathrm{a}, \mathrm{b})$ combinations can get different GLCMs.

3) Features based on Gray-Level Co-occurrence Matrix

Based on GLCM, we select some characteristic quantities to reflect the condition of the matrix[32], as shown:

Energy:

$$
f_{1}=\sum_{i=0}^{L-1} \sum_{j=0}^{L-1} P_{i j}^{2}
$$

It reflects the uniformity of the image gray scale distribution and the texture roughness. When the $P_{i j}$, value distribution is more concentrated, the energy is larger. When the $P_{i j}$ value distribution is more dispersed, the energy is smaller.

Correlation:

$$
f_{2}=\frac{1}{\sigma_{x} \sigma_{y}} \sum_{i=0}^{L-1} \sum_{j=0}^{L-1}\left(i-\mu_{x}\right)\left(j-\mu_{y}\right) P_{i j}
$$

Where:

$$
\begin{gathered}
\mu_{x}=\sum_{i=0}^{L-1} i \sum_{j=0}^{L-1} P_{i j} \\
\mu_{y}=\sum_{j=0}^{L-1} j \sum_{i=0}^{L-1} P_{i j} \\
\sigma_{x}^{2}=\sum_{i=0}^{L-1}\left(i-\mu_{x}\right)^{2} \sum_{j=0}^{L-1} P_{i j} \\
\sigma_{y}^{2}=\sum_{j=0}^{L-1}\left(j-\mu_{y}\right)^{2} \sum_{i=0}^{L-1} P_{i j}
\end{gathered}
$$

It measures the similarity of the matrix elements in the row and column directions, that is, the local gray correlation in the image is reflected. When the matrix element gap is small, the correlation value is large. When the matrix elements differ greatly, the correlation value is small.

Entropy:

$$
f_{3}=-\sum_{i=0}^{L-1} \sum_{j=0}^{L-1} P_{i j} \log _{2} P_{i j}
$$

Entropy is a measure of the amount of information of an image, which represents the complexity of the texture. If the distribution of $P_{i j}$ is relatively uniform, then the entropy is large. If the distribution of $P_{i j}$ is more concentrated, the entropy is smaller.

Contrast:

$$
f_{4}=\sum_{i=0}^{L-1} \sum_{j=0}^{L-1}|i-j|^{2} P_{i j}
$$

For coarse textures, contrast is small. For fine textures, contrast is large.

In this paper, we take $(\mathrm{a}, \mathrm{b})$ of 4 combinations $(1,0),(-1,1)$, $(-1,0)$ and $(-1,1)$ to generate 4 GLCMs for each sky image, and the corresponding energy, correlation, entropy and contrast mean values are calculated as the four dimensional texture feature vectors of each image. Then, we take the mean of the feature vectors of two adjacent images as their common feature vectors and take them as samples of pattern classification.

\section{4) K-means clustering}

The k-means clustering algorithm is a widely used unsupervised clustering algorithm. It iterative partitioning minimizes the sum, over all clusters, of the within-cluster sums of point-to-cluster-centroid distances. The squared Euclidean distance is used in this approach [33]. Compared with other clustering algorithms, it has the advantages of simple and efficient, low time and space complexity. Therefore, this paper chooses k-means to cluster the cloud.

The k-means clustering method is approached to achieve the classification according to the feature vectors obtained in the previous step. However, the number of clusters $\mathrm{K}$ of $\mathrm{k}$-means needs to be specified by humans. When $\mathrm{K}$ is too small, the classification model cannot distinguish all the modes. And when $\mathrm{K}$ is too large, the classification model is easy to overfit (cannot effectively classify new samples). It is unrealistic to artificially obtain the optimal $\mathrm{K}$ (simulation test on a large amount of historical data). So we use the optimal cluster number criterion in the Matlab toolbox.

Compared with other criterions, the Calinski-Harabasz $(\mathrm{CH})$ is simple, easy to understand and widely used, so we use the $\mathrm{CH}$ criterion to determine the optimal $\mathrm{K}$. 


$$
C H=\frac{\sum_{i=1}^{K}\left\|x_{i}-\bar{x}\right\|}{\sum_{i=1}^{K} \sum_{p \in C_{i}}\left\|p-x_{i}\right\|}
$$

Where: $p$ is the sample point in the class $C_{i}, x_{i}$ is the cluster-

ing center of $\mathrm{C}$, and $\bar{x}$ is the sample mean. Obviously, the larger the value of $\mathrm{CH}$, the more optimized the number of clusters. In this paper, in order to visually represent the change in the value of the criterion, we take $\mathrm{CH}$ to the inverse. In this way, we need to select the corresponding $\mathrm{K}$ value when the value of criterion is small adequately and the decline trend becomes slow enough.

\section{B. The Three Submethods of Combined Calculation Modeling}

We explain briefly the principles of the three sub methods mentioned above firstly, and then introduce the so-called combined calculation modeling.

\section{1) Block-Matching algorithm}

The rationale of Block-Matching is that every last image in the frame sequence is subdivided into sub blocks, and afterward, compares all the candidate sub blocks in a given search area of the current frame to one sub block of the previous frame. Owning to the principle of minimum difference of accumulated gray value, the sub block which resembles the previous sub block most is found, namely matching block. In this way, the displacement between the previous block and the matching block is the motion vector of the block.

According to literature [22] and [34], the following four formulas are used to measure the difference in gray values between the two images:

Correlation coefficient:

$$
\begin{gathered}
R_{1}=\frac{\sum_{i=1}^{M} \sum_{j=1}^{N}\left[f_{1}\left(x_{i}, y_{j}\right)-\overline{f_{1}}\right]\left[f_{2}\left(x_{i}, y_{j}\right)-\overline{f_{2}}\right]}{\sqrt{\sum_{i=1}^{M} \sum_{j=1}^{N}\left[f_{1}\left(x_{i}, y_{j}\right)-\overline{f_{1}}\right]^{2}} \sqrt{\sum_{i=1}^{M} \sum_{j=1}^{N}\left[f_{2}\left(x_{i}, y_{j}\right)-\overline{f_{2}}\right]^{2}}} \\
R_{2}=\frac{\sum_{i=1}^{M} \sum_{j=1}^{N} f_{1}\left(x_{i}, y_{j}\right) f_{2}\left(x_{i}, y_{j}\right)}{\sqrt{\sum_{i=1}^{M} \sum_{j=1}^{N} f_{1}\left(x_{i}, y_{j}\right)^{2}} \sqrt{\sum_{i=1}^{M} \sum_{j=1}^{N} f_{2}\left(x_{i}, y_{j}\right)^{2}}}
\end{gathered}
$$

Variance:

$$
R_{3}=\frac{1}{M N} \sum_{i=1}^{M} \sum_{j=1}^{N}\left[f_{1}\left(x_{i}, y_{j}\right)-f_{2}\left(x_{i}, y_{j}\right)\right]^{2}
$$

Sum of grey value differences:

$$
R_{4}=\sum_{i=1}^{M} \sum_{j=1}^{N}\left|f_{1}\left(x_{i}, y_{j}\right)-f_{2}\left(x_{i}, y_{j}\right)\right|
$$

Where: $f_{1}\left(x_{i}, y_{j}\right)$ and $f_{2}\left(x_{i}, y_{j}\right)$ are the gray function of two images $I_{1}$ and $I_{2} . I_{1}$ is the previous frame image and $I_{2}$ is the next frame image. $\bar{f}_{1}$ and $\overline{f_{2}}$ are the gray average, $M^{*} N$ is the size of images, that is to say, it is the size of the corresponding gray scale matrixes.

Obviously, when $R_{1}$ and $R_{2}$ are at their maximum, or $R_{3}$ and $R_{4}$ are at their minimum, the two images are the most similar.

However, the calculation of the original Block-Matching algorithm is very large, so that it is difficult to meet the requirements of real-time computing. In order to reduce the workload, a Block-Matching Fast Fourier Transform algorithm [35] is widely used. In this method, the digital image is regarded as a sequence of discrete two-dimensional signal field with time, and the computation speed is greatly improved by using the method of signal analysis.

To sum up, Block-Matching is a widely used and very basic method for cloud velocity calculation. In the combined calculation modeling below, we used the corresponding convenient Particle Image Velocimetry toolbox in Matlab to accomplish the mission of cloud speed calculation.

\section{2) Optical Flow algorithm}

The optical flow is the "instantaneous velocity $(\mathrm{u}, \mathrm{v})$ " of the moving object in the observed image plane. As for Optical Flow algorithm, its essence is to establish the optical flow constraint equation in line with the truth of image intensity conservation. By solving the equation above, the velocity parameters can be acquired.

There are three premise hypotheses of optical flow:

Hypothesis 1: The gray levels of the corresponding pixels in adjacent images are constant.

Hypothesis 2: The displacement of the target in the adjacent image is relatively small.

Hypothesis 3: A pixel has the same displacement as the pixel in the neighborhood.

The first step: according to hypothesis 1 , we can get that:

$$
f(x, y, t)=f(x+d x, y+d y, t+d t)
$$

Where: $f(x, y, t)$ is the gray function of the image. And $(x, y)$ represent the pixel coordinates, and $t$ represents the time.

Take Taylor decomposition on the right side of the formula, we can get that: 


$$
\begin{gathered}
f(x+d x, y+d y, t+d t)=f(x, y, t)+\frac{\partial f}{\partial x} d x \\
+\frac{\partial f}{\partial y} d y+\frac{\partial f}{\partial t} d t+R_{1}(x, y, t)
\end{gathered}
$$

Where:

$$
R_{1}(x, y, t)=o\left(\sqrt{(d x)^{2}+(d y)^{2}+(d t)^{2}}\right)
$$

The second step: based on the assumption that 2, the remainder can be neglected, so we can get that:

$$
\begin{gathered}
f(x+d x, y+d y, t+d t)=f(x, y, t)+ \\
\frac{\partial f}{\partial x} d x+\frac{\partial f}{\partial y} d y+\frac{\partial f}{\partial t} d t
\end{gathered}
$$

Let $f_{x}=\frac{\partial f}{\partial x}, f_{y}=\frac{\partial f}{\partial y}, f_{t}=\frac{\partial f}{\partial t}, u=\frac{d x}{d t}$ and $v=\frac{d y}{d t}$, we can get that:

$$
f_{x} u+f_{y} v=-f_{t}
$$

Written in matrix form:

$$
\left[f_{x} f_{y}\right]\left[\begin{array}{l}
u \\
v
\end{array}\right]=-f_{t}
$$

However, two unknown quantities $(\mathrm{u}, \mathrm{v})$ cannot be obtained by one equation.

The third step: according to hypothesis 3 , if the displacement of $\mathrm{n}$ points in the neighborhood is the same, then the following $n(n>2)$ equations are obtained:

$$
\left[\begin{array}{cc}
f_{x 1} & f_{y 1} \\
f_{x 2} & f_{y 2} \\
\mathrm{M} & \mathrm{M} \\
f_{x n} & f_{y n}
\end{array}\right]\left[\begin{array}{l}
u \\
v
\end{array}\right]=-\left[\begin{array}{c}
f_{t 1} \\
f_{t 2} \\
\mathrm{M} \\
f_{t n}
\end{array}\right]
$$

The fourth step: using the least squares method to solve the above linear equations, we can get $(\mathrm{u}, \mathrm{v})$ :

$$
\text { Let }\left[\begin{array}{cc}
f_{x 1} & f_{y 1} \\
f_{x 2} & f_{y 2} \\
\mathrm{M} & \mathrm{M} \\
f_{x n} & f_{y n}
\end{array}\right]=A,\left[\begin{array}{l}
u \\
v
\end{array}\right]=x \text {, and }-\left[\begin{array}{c}
f_{t 1} \\
f_{t 2} \\
\mathrm{M} \\
f_{t n}
\end{array}\right]=b \text {, and the above }
$$

formula is simplified as:

$$
A x=b
$$

Let the optimization index be the sum of the least squares of errors. That is, the variable $x$ is obtained when $\|A x-b\|^{2}$ reaches the minimum value.

In the combined calculation modeling below, we use LK optical flow method to accomplish the mission of cloud speed calculation.

\section{3) SURF feature matching algorithm}

When it comes to feature matching, you need to find the feature points that correspond to each other in the two successive images, and then calculate the distance between them. Common feature extraction methods include corner features (such as Harris operator [36]), line features (image edge detection), local area characteristics (speckle features) and invariant features (scale invariant features) and so on.

In this paper, we select the SURF feature operator [37] for image matching. It can extract the feature points better in the case of illumination change and image change, and the scale invariance is better than Harris operator, the time complexity is better than the SIFT operator. The construction of the SURF operator is very complicated, and we only make a brief explanation.

The first step: detect and locate feature points.

SURF uses Hessian matrix $H(x, \sigma)$ to detect feature points:

$$
H(x, \sigma)=\left[\begin{array}{l}
L_{x x}(x, \sigma) L_{x y}(x, \sigma) \\
L_{x y}(x, \sigma) L_{y y}(x, \sigma)
\end{array}\right]
$$

Where: $x$ is the characteristic point coordinate, $\sigma$ is the scale, $L_{x x}(x, \sigma)$ is the convolution of the image with the Gaussian second order differential $\frac{\partial^{2}}{\partial x^{2}} g(\sigma)(g(\sigma)$ is the Gaussian function, the other meaning is similar).

In order to reduce the running time of the algorithm, the Gaussian template is simplified as a rectangular region box filter, and $D_{x x}, D_{y y}$ and $D_{x y}$ are used to denote the convolution of the box and the image. The determinant of the Hessian matrix can be approximated as follows:

$$
\Delta(H)=D_{x x} D_{y y}-\left(0.9 D_{x y}\right)^{2}
$$

The scale invariance of the SURF algorithm mainly depends on finding the feature points at different scales. The scale space is divided by the Octaves, and each group represents the response graph of the incremental filtering template and the image convolution. The first set of scale spaces are $9 * 9,15 * 15,21 * 21$, $27 * 27$; the other groups increase similar, but the increments are doubled, 6, 12 and 24, respectively. In order to cover all the scales, there is overlap between the group and the group. 
In order to locate the feature points, the extreme suppression method in the 3D space is used to find the extreme points. That is, the Hessian matrix feature points are compared with the other 26 values of the $3 * 3 * 3$ scale space centered on that point, and only if the point is larger or smaller than the other values, it is the local extreme point. Then, interpolation is made in the scale space and the image space to obtain the final feature point location and the scale value.

The second step: generate the feature point description operator.

In order to make the feature have better rotation invariance, it is necessary to assign the main direction to each feature point.

Firstly, in the circular area with the feature point as the center and the scale of 6 times the radius, the Haar wavelet response of each point is calculated.

Then, add all Haar wavelet $d x$ and $d y$ in the range of 60 degrees to form a new vector $\left(m_{w}, \theta_{w}\right)$ :

$$
\begin{aligned}
& m_{w}=\sum d x+\sum d y \\
& \theta_{w}=\arctan \left[\frac{\sum d x}{\sum d y}\right]
\end{aligned}
$$

The whole circular area is traversed by $0.2 \mathrm{rad}$ in length and the longest vector represents the main direction of the feature point.

Afterward, take the feature point as the center, rotate the coordinate axis to the main direction, select the square area of the $20 s^{*} 20 \mathrm{~s}$ ( $\mathrm{s}$ is the scale of the feature points), and divide it into $4 * 4$, i.e. 16 square sub windows, each side window is 5 s.

After that, the Gaussian weighted $d x$ and $d y$ along the main direction and perpendicular to the main direction are obtained using a Haar wavelet template with a scale of $2 \mathrm{~s}$.

Finally, in each square, the responses of the Haar wavelet in the $\mathrm{x}$ and $\mathrm{y}$ directions are summed (the $\mathrm{x}$ direction is perpendicular to the main direction and the $y$ direction is parallel to the main direction) to form a four dimensional vector:

$$
v=\left[\begin{array}{llll}
\sum d x & \sum|d x| & \sum d y & \sum|d y|
\end{array}\right]
$$

In addition, we normalize it to form $16 * 4$, i.e. 64 dimensional SURF description operator. In addition, the Haar wavelet response itself has brightness invariance, and the contrast invariance can be achieved by normalization of feature vectors. In general, it improves the anti-interference ability.

\section{Combined Calculation Modeling}

Particle Swarm Optimization (PSO) is similar to Genetic Algorithm (GA) and is also an iterative optimization algorithm. The system is initialized as a set of random solutions, and the optimal values are searched iteratively. Compared with GA, it is easier to achieve and does not need to adjust too many parameters [38]-[39]. At present, it has been widely used in function optimization, neural network training, fuzzy system control and other applications of GA.

In this paper, we use PSO to assign different weights to each method in the combined calculation modeling on the basis of diverse cloud classes. In other words, the combined calculation modeling is built. As shown in (27):

$$
V_{\text {Cloud }}=a V_{B M}+b V_{O F}+c V_{S U R F}
$$

Where $V_{C \text { loud }}$ indicates the final cloud motion speed, $V_{B M}, V_{O F}$ and $V_{S U R F}$ indicate the cloud motion speed calculated by BlockMatching, Optical Flow, and SURF feature matching algorithm respectively, a, b and c are weights obtained by PSO and they vary with the cloud classes.

Here, we use the correlation coefficient $R_{1}$ (as shown in Formula 10) as the optimization function, and the reciprocal of $R_{1}$ as the fitness function of the particles in the PSO.

$$
R_{1}=\frac{\sum_{i=1}^{M} \sum_{j=1}^{N}\left[f_{1}\left(x_{i}, y_{j}\right)-\overline{f_{1}}\right]\left[f_{2}\left(x_{i}, y_{j}\right)-\overline{f_{2}}\right]}{\sqrt{\sum_{i=1}^{M} \sum_{j=1}^{N}\left[f_{1}\left(x_{i}, y_{j}\right)-\overline{f_{1}}\right]^{2}} \sqrt{\sum_{i=1}^{M} \sum_{j=1}^{N}\left[f_{2}\left(x_{i}, y_{j}\right)-\overline{f_{2}}\right]^{2}}}
$$

Mind: $f_{1}(x, y)$ and $f_{2}(x, y)$ represent part of shift images. And they are based on $\left(x_{0}, y_{0}\right)$ and cut from the original images.

By the way, the specific practice of "cutting" is as follows.

It is assumed that $V_{\text {Cloud }}=\left(x_{0}, y_{0}\right)=(3,5)$ is obtained from a certain algorithm. Then for the previous frame image, we need to cut out the pixels in the first 5 rows and the last 3 columns to get the above $f_{1}(x, y)$. And for the next frame image, we need to cut out the pixels in the first 3 columns and the last 5 rows to get the above $f_{2}(x, y)$. As shown in the following Fig.2, the gray part of shift images is cut off, while the blue part is the corresponding $f_{1}(x, y)$ and $f_{2}(x, y)$.

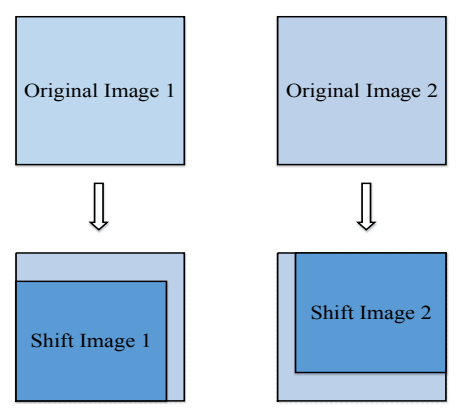

Fig.2 Cutting Process 
Obviously, the range of $R_{1}$ is $[-1,1]$ and the larger the value of $R_{1}$, the more similar the two images (that is to say the $V_{C l o u d}$ calculated is more accurate). In other words, the smaller the fitness of the particle, the better the particle is.

\section{CASE STUDY}

\section{A. Data description}

We use sky images provided by Yunnan Electric Power Research Institute $\left(25^{\circ} 09^{\prime} 53.35^{\prime}\right.$ N, $102^{\circ} 39^{\prime} 32^{\prime}$ E) as the experimental sample. These photos were taken in August 2015 and at intervals of 1 minute. The resolution of these images is $256 * 256$. Among them, 300 images were selected as training samples and 200 images were used as testing samples.

The hardware parameters used for simulation test are as follows: i7-6700HQ(CPU), GTX960M 4G(GPU), $16 \mathrm{G}$ DDR4(RAM), Windows 10(OS), and Matlab 2015b (computing platform).

The toolboxes based on Matlab platform: Histogram Equalization, Gray-level Co-occurrence Matrix, Calinski-Harabasz, Kmeans, Particle Image Velocimetry, Piotr's Computer Vision, and Computer Vision System.

\section{B. Results and Comparison with Other Classical Algorithms}

\section{1) Pattern Classification of Clouds}

The value of the criterion changes as the number of clusters changes as shown in Fig. 3.

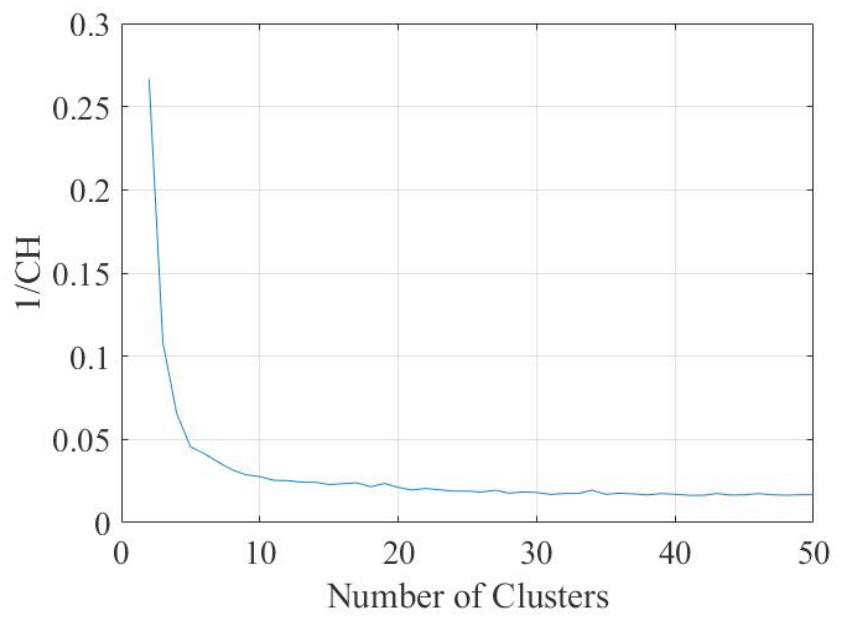

Fig.3 The trend of index when $\mathrm{K}$ is optimized

Obviously, when $\mathrm{K}=10$, the value of criterion is small adequately and the decline trend becomes slow enough. That is to say, the K-means clustering method is used to divide the images into 10 categories.

\section{2) Combined Calculation Modeling}

In this section, we use PSO to optimize the three weights a, $\mathrm{b}$ and $\mathrm{c}$. The population size is set to 100 and the number of evolution times is also set to 100 . The trend of optimal fitness with the number of evolution times in the process of weight optimization for a pair of continuous images in a certain class is shown in Fig. 4.

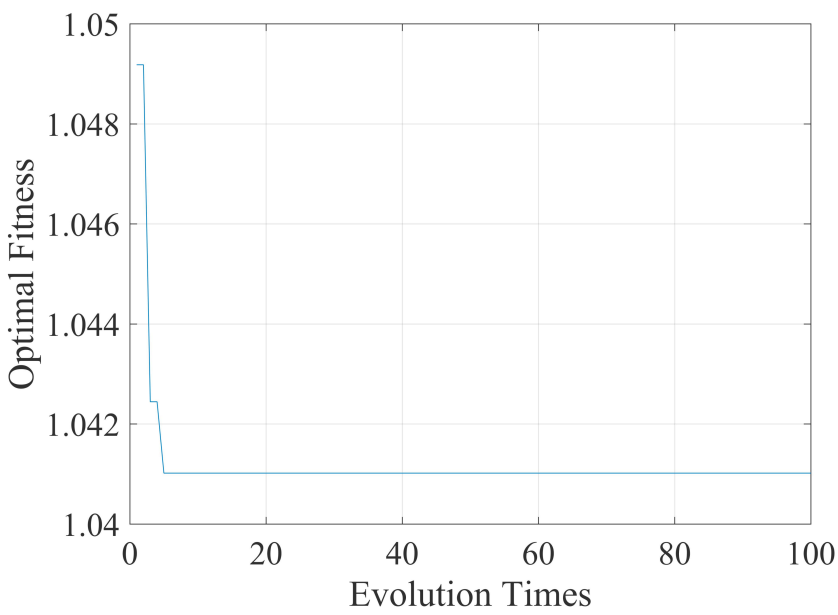

Fig.4 The trend of index when weights are optimized

Finally, we obtain the corresponding 10 sets of weights $a, b$, and $\mathrm{c}$. By the way, each set of weights here is the average value of the weights obtained by the PSO optimization of all the images in the corresponding class. That is to say, for different types of sky images, we have established 10 corresponding models for cloud velocity calculation. The specific numerical of the weights is listed in Table 1.

TABLE I. THE SPECIFIC WEIGHTS OF THE 10 CLASSES

\begin{tabular}{cccc}
\hline Class & $\mathbf{a}$ & $\mathbf{b}$ & $\mathbf{c}$ \\
\hline 1 & 0.5606 & 0.5161 & 0.3416 \\
2 & 0.6999 & 0.7571 & 0.2551 \\
3 & 0.7821 & 0.7043 & 0.3924 \\
4 & 0.9553 & 0.9203 & 0.8058 \\
5 & 0.5285 & 0.6277 & 0.5601 \\
6 & 0.1107 & 0.7609 & -0.7569 \\
7 & 0.5521 & 0.6711 & 0.7862 \\
8 & 0.5528 & 0.5490 & 0.5006 \\
9 & -0.1928 & -0.3885 & 0.7683 \\
10 & 0.4425 & 0.6424 & 0.5698 \\
\hline
\end{tabular}

Next, we will test the proposed modeling in this paper using test images and compare the results with those of the three traditional methods. Of course, for new image sequence, we need to extract the feature vectors described above and calculate the Euclidean distance between them and the above 10 clustering 
centers to classify images and then use the corresponding computational modeling for cloud velocity calculation. As mentioned above, we use $R_{1}$ (Formula 10), $R_{3}$ (Formula 12) and $R_{4}$ (Formula 13) to evaluate the accuracy of the algorithm. The accuracy of each algorithm in different cloud classes is shown in the graphs and tables below, and the highest precision corresponding to each class is shown in bold. For simplicity, the Block-Matching is abbreviated as BM, the Optical Flow is abbreviated as OF, and the SURF feature is abbreviated as SURF.

As shown in the following Table 2 to $5 \mathrm{~s}$ and Figure 5 to 12 .

TABLE II. $\quad R_{1}$ VALUE OF EACH ALGORITHM

\begin{tabular}{ccccc}
\hline \multirow{2}{*}{ Class } & \multicolumn{5}{c}{$R_{1}$} \\
\cline { 2 - 5 } & $\boldsymbol{P C P O \boldsymbol { W }}$ & $\boldsymbol{B M}$ & $\boldsymbol{O F}$ & $\boldsymbol{S U R \boldsymbol { F }}$ \\
\hline 1 & $\mathbf{0 . 9 4 9 1}$ & 0.9441 & 0.9302 & 0.8818 \\
2 & $\mathbf{0 . 8 9 9 3}$ & 0.8578 & 0.898 & 0.7571 \\
3 & $\mathbf{0 . 9 6 9 9}$ & 0.9696 & 0.938 & 0.8561 \\
4 & $\mathbf{0 . 9 6 7 1}$ & 0.9573 & 0.9466 & 0.9334 \\
5 & $\mathbf{0 . 9 6 9 4}$ & 0.9579 & 0.9595 & 0.9592 \\
6 & $\mathbf{0 . 8 5 1 1}$ & 0.673 & 0.7047 & 0.5659 \\
7 & $\mathbf{0 . 9 3 2 2}$ & 0.8923 & 0.9102 & 0.9192 \\
8 & $\mathbf{0 . 9 4 5 7}$ & 0.9455 & 0.9425 & 0.9404 \\
9 & $\mathbf{0 . 8 7 7 1}$ & 0.7712 & 0.6288 & 0.7291 \\
10 & $\mathbf{0 . 8 8 9 2}$ & 0.8325 & 0.8693 & 0.8552 \\
\hline
\end{tabular}

TABLE III. $\quad R_{3}$ VALUE OF EACH ALGORITHM

\begin{tabular}{ccccc}
\hline \multirow{2}{*}{ lass } & \multicolumn{4}{c}{$R_{3}$} \\
\cline { 2 - 5 } & $\boldsymbol{P C P O} \boldsymbol{W}$ & $\boldsymbol{B M}$ & $\boldsymbol{O F}$ & $\boldsymbol{S} \boldsymbol{U} \boldsymbol{F}$ \\
\hline 1 & $\mathbf{7 5 . 7 8 8 9}$ & 91.9612 & 94.3058 & 143.5700 \\
2 & $\mathbf{1 3 7 . 1 4 2 9}$ & 164.3760 & 137.9458 & 228.7062 \\
3 & $\mathbf{3 2 . 2 2 1 7}$ & 37.4204 & 113.1213 & 179.3760 \\
4 & $\mathbf{3 9 . 4 4 8 4}$ & 49.8854 & 68.8821 & 88.2217 \\
5 & $\mathbf{3 7 . 8 5 6 9}$ & 48.1127 & 44.5527 & 46.8884 \\
6 & $\mathbf{1 9 1 . 3 4 4 0}$ & 341.7697 & 328.2218 & 897.2105 \\
7 & $\mathbf{8 9 . 9 7 5 8}$ & 149.1241 & 99.4588 & 139.1773 \\
8 & $\mathbf{8 5 . 9 6 1 2}$ & 86.5472 & 89.5472 & 91.5667 \\
9 & $\mathbf{1 9 2 . 1 4 2 9}$ & 590.5572 & 771.9547 & 358.9951 \\
10 & $\mathbf{1 2 7 . 1 4 4 9}$ & 185.3224 & 162.5534 & 152.5531 \\
\hline
\end{tabular}

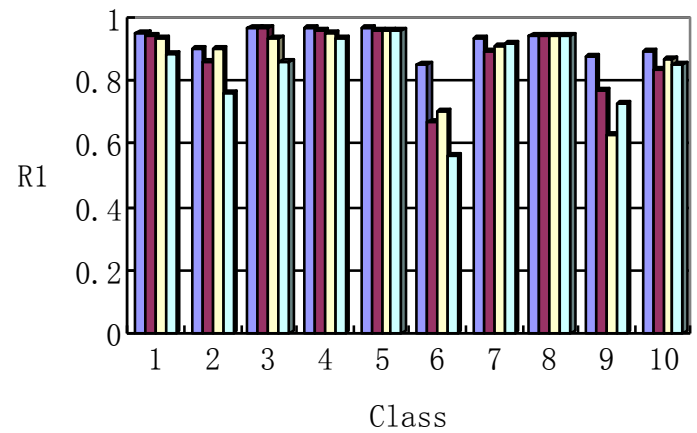

$\square$ PCPOW $\square$ BM $\square$ OF $\square$ SURF

Fig.5 $R_{1}$ value of each algorithm

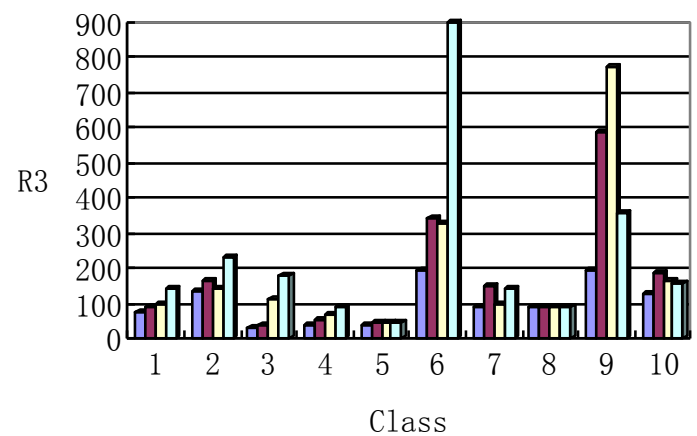

$\square$ PCPOW $\square$ BM $\square$ OF $\square$ SURF

Fig.6 $R_{3}$ value of each algorithm

TABLE IV. $\quad R_{4}$ VALUE OF EACH ALGORITHM

\begin{tabular}{ccccc}
\hline \multirow{2}{*}{ Class } & \multicolumn{4}{c}{$R_{4}$} \\
\cline { 2 - 5 } & $\boldsymbol{P C P O W}$ & $\boldsymbol{B M}$ & $\boldsymbol{O F}$ & $\boldsymbol{S U R \boldsymbol { F }}$ \\
\hline 1 & $\mathbf{3 5 7 1 0 3}$ & 392214 & 451015 & 501201 \\
2 & $\mathbf{4 7 1 1 0 1}$ & 510212 & 482101 & 634478 \\
3 & $\mathbf{2 9 4 2 1 8}$ & 300011 & 423014 & 530421 \\
4 & $\mathbf{3 0 1 2 9 8}$ & 384214 & 397859 & 432148 \\
5 & $\mathbf{2 9 4 5 7 8}$ & 378784 & 367984 & 312657 \\
6 & $\mathbf{5 0 2 0 1 8}$ & 685889 & 712304 & 981034 \\
7 & $\mathbf{4 3 2 0 1 5}$ & 521457 & 475334 & 453157 \\
8 & $\mathbf{3 6 1 3 2 7}$ & 359999 & 369759 & 376037 \\
9 & $\mathbf{4 8 6 5 2 9}$ & 574478 & 758862 & 510024 \\
10 & $\mathbf{4 2 2 3 6 5}$ & 539998 & 450031 & 538421 \\
\hline
\end{tabular}




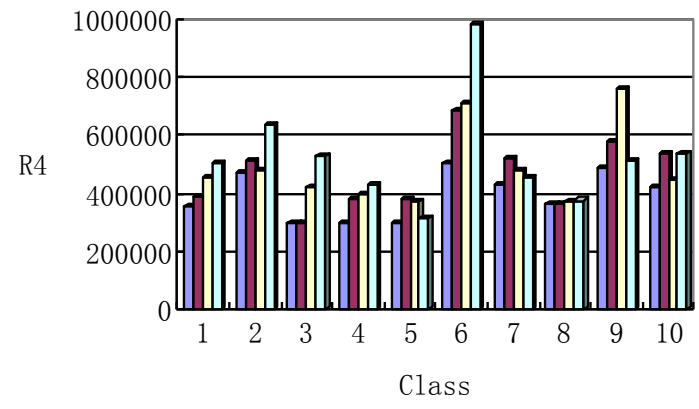

$\square$ PCPOW $\square$ BM $\square$ OF $\square$ SURF

Fig.7 $R_{4}$ value of each algorithm

It can be seen that the proposed combined calculation modeling in this paper has higher accuracy than the other three traditional methods. Especially, the calculation precision of SURF feature matching algorithm and Optical Flow algorithm are very low in class 6 and in class 9 separately, but our method can still maintain a relatively high accuracy.

Then, we choose several extreme sky images to specify the limitations of the three single methods.

The sky images in Fig. 8 belong to the class 6 . In the images, the most similar SURF feature points are marked with green circles. Obviously, the result of the SURF feature matching algorithm indicates that the cloud moves toward the lower left, but in fact the cloud moves toward the lower right. In other words, the two SURF feature points have been mismatched.

Because the texture information of the image is not rich enough, the texture features of each area have little difference, which results in no obvious difference in the 64 dimensional SURF description operator, so the mismatch phenomenon is easy to occur.
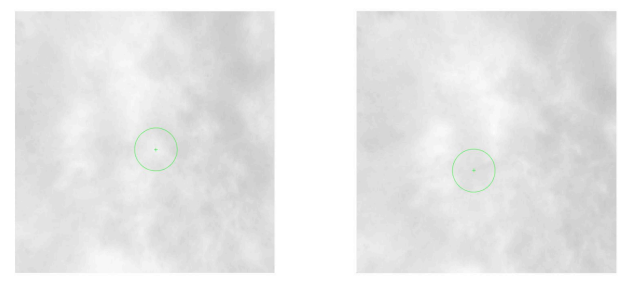

Fig.8 Sky images with flat texture

The sky images shown in Fig. 9 belong to the class 9 and the following pictures show the displacement vector of each pixel in the images. Obviously, the displacement distribution of pixels in the graph is discrete. In other words, the pixels of cloud lack a "common" displacement vector, so when we take the mean value of the displacements of these pixels as the displacement of the cloud, the accuracy of the calculation results will be reduced.
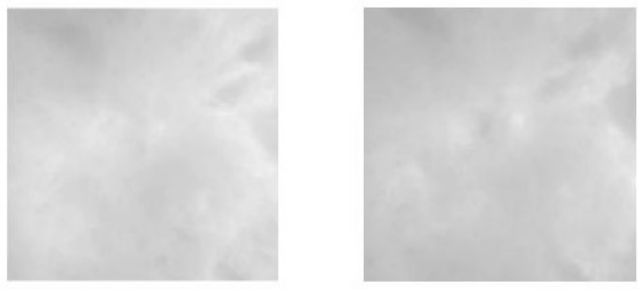

Fig.9 Sky images with uneven illumination

Because the brightness of the corresponding positions of the two images is generally different, this leads to the "Hypothesis 1 " is no longer satisfied and the calculation error is produced.
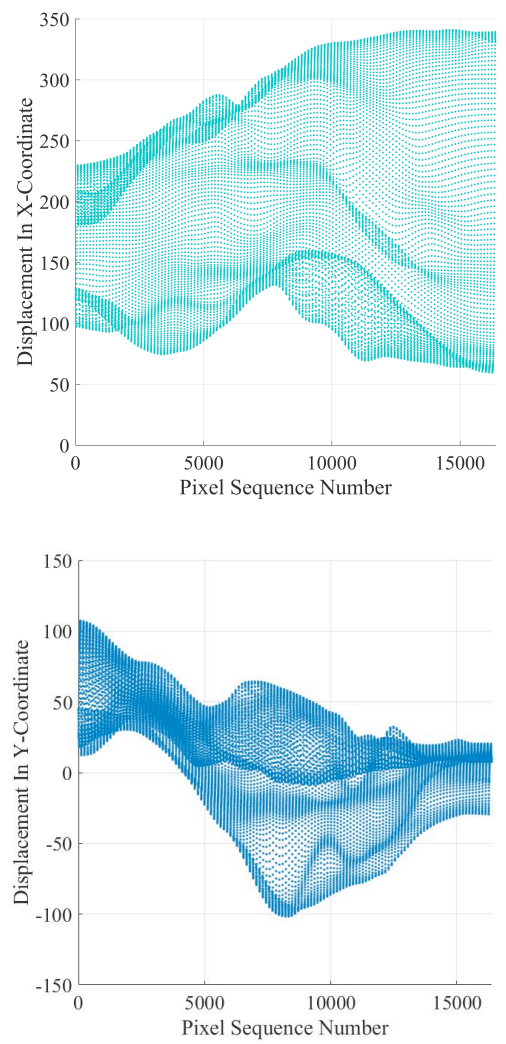

Fig.10 Displacement vector of pixels in the direction of $\mathrm{X}$ and $\mathrm{Y}$

The sky images shown in Fig. 11 also belong to the class 9 . Based on the process of Block-Matching algorithm, we divide the image into many sub blocks, then find the most similar blocks according to the principle of gray similarity. Obviously, the sub blocks marked by the red box in the two images are identified as the most similar blocks by the algorithm. In other 
words, due to the strong deformation of the cloud, the situation of mismatching occurs.
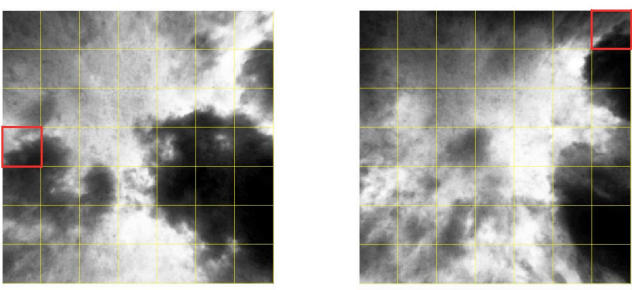

Fig.11 Sky images with strong deformation

Next, in order to prove the validity of "pattern classification" in this method, we use the same weights $(a=b=c=1 / 3)$ to calculate cloud motion vectors for the above 10 classes of cloud. Under the two sets of weight coefficients, the accuracy of the $\mathrm{R} 1$ calculation is shown in Table 5 and Fig. 12.

TABLE V. COMPARISON OF ACCURACY OF CLASSIFICATION OR NOT

\begin{tabular}{ccc}
\hline \multirow{2}{*}{ Class } & \multicolumn{2}{c}{$R_{1}$} \\
\cline { 2 - 3 } & The different weights & The same weights \\
\hline 1 & 0.9491 & 0.4494 \\
2 & 0.8993 & 0.5136 \\
3 & 0.9699 & 0.5264 \\
4 & 0.9671 & 0.597 \\
5 & 0.9694 & 0.9431 \\
6 & 0.8511 & 0.8389 \\
7 & 0.9322 & 0.8529 \\
8 & 0.9457 & 0.8776 \\
9 & 0.8771 & 0.8519 \\
10 & 0.8892 & 0.887 \\
\hline
\end{tabular}

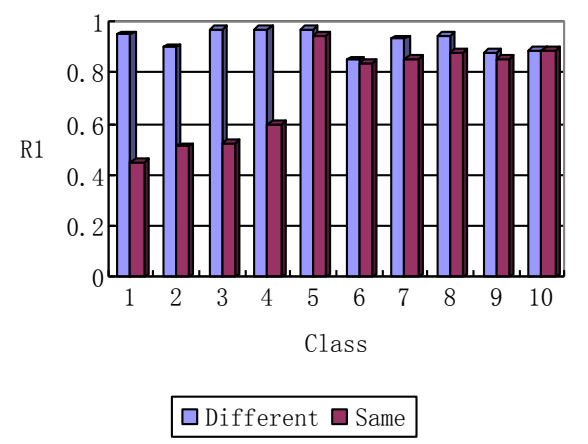

Fig.12 Comparison of accuracy of classification or not
It can be seen that if different computing strategies are not adopted for different classes of sky images, the overall accuracy of the testing samples will be greatly reduced. This shows that sky images have different classes and we cannot obtain high precision calculation results by a single computing strategy.

\section{Discussion}

\section{1) Cross-validation}

In order to avoid the contingency of the simulation results and verify the robustness of the proposed model, we applied cross-validation method to further develop the simulation.

For the 500 sample images selected in the section A, random selection is performed in a 3:2 ratio for each class of sky image set to generate new training and testing sets and we get a total of 300 training samples and 200 testing samples. To ensure the validity of the cross-validation test, the class distribution in newly generated training and testing sets are the same as that in section A. Here we make 10 random selections in the same way.

Then, we use the above samples to train and test the model, and show the average calculation accuracy R1 of the testing images in Table 6.

TABLE VI. ACCURACY OF CROSS-VALIDATION

\begin{tabular}{cc}
\hline Number of cross-validation & $R_{1}$ \\
\hline 1 & 0.9207 \\
2 & 0.9231 \\
3 & 0.9344 \\
4 & 0.9229 \\
5 & 0.9277 \\
6 & 0.9199 \\
7 & 0.9381 \\
8 & 0.9322 \\
9 & 0.9276 \\
10 & 0.9351 \\
\hline
\end{tabular}

Obviously, it can be seen that in the above 10 crossvalidations, the average accuracies are similar, which proves that the model is highly robust.

\section{2) Rolling simulation}

Theoretically, a rolling simulation is necessary to simulate the actual situation for the application of this model. However, due to the limitation of available samples, the results of rolling simulation have little practical significance. Here an example is given to illustrate this.

We select the first 50 and 100 consecutive images respectively as training samples, and classify them according to the above process. The classification results are shown in Table 7 and 8 .

Obviously, compared with the 300 randomly selected training samples in the section A, the first 50 or 100 consecutive training samples contain too few motion patterns. In other words, they 
cannot provide a complete pattern recognition library to fully reflect different patterns and rules.

TABLE VII. CLASSIFICATION RESULTS FOR THE FIRST 50 IMAGES

\begin{tabular}{cc}
\hline Class & Amount of image pairs \\
\hline 1 & 9 \\
2 & 0 \\
3 & 11 \\
4 & 7 \\
5 & 0 \\
6 & 0 \\
7 & 22 \\
8 & 0 \\
9 & 0 \\
10 & 0 \\
\hline
\end{tabular}

TABLE VIII. CLASSIFICATION RESULTS FOR THE FIRST 100 IMAGES

\begin{tabular}{cc}
\hline Class & Amount of image pairs \\
\hline 1 & 18 \\
2 & 0 \\
3 & 15 \\
4 & 9 \\
5 & 0 \\
6 & 14 \\
7 & 26 \\
8 & 0 \\
9 & 0 \\
10 & 17 \\
\hline
\end{tabular}

Then, we use the 50 or 100 training samples up-to the current minute to calculate next minute and roll the calculate origin forward until providing calculation for all the testing samples. And show the average calculation accuracy R1 of the testing images in Table 9.

TABLE IX. ACCURACY OF ROLLING SIMULATION

\begin{tabular}{cc}
\hline Capacity of training sample & $R_{1}$ \\
\hline 50 & 0.7747 \\
100 & 0.8125 \\
\hline
\end{tabular}

Obviously, because the motion patterns of some testing samples cannot be recognized correctly, the accuracy of the calculation results is low. In summary, rolling prediction has limitations when the sample is insufficient.

\section{CONCLUSION}

As the cloud in sky images has different motion patterns and the existing cloud speed calculation method has poor robustness, it is necessary to classify the sky images and then establish corresponding combination optimization models. In this paper, through the analysis of the three current mainstream methods
(Block-Matching, Optical Flow and SURF feature matching algorithm), we proposed a pattern classification and PSO optimal weight-based sky images cloud motion speed calculation method for solar PV power forecasting (PCPOW). Actual sky image data has been utilized to verify the effectiveness of the proposed method. By case study and the comparing between the other three traditional methods, the result proves the method could overcome the weaknesses of the traditional single method in the applicable scope and it is a more universal modeling suitable for most cloud scenes. In the future study based on the previous works [40]-[44], we should pay attention to the preprocessing of sky image, not only to ensure the operation speed, but also to ensure the image information is not lost too much. In addition, how to extract features so as to achieve more efficient classification of images is also another focus. Furthermore, to verify the effectiveness of the proposed method in the final combining solar PV power forecasting model with the consideration of other impact factors such as PV module temperature [16] will be conducted, which can help to address the household characteristics impacts on residential electricity consumption patterns [45] and improve the accuracy of the customer base line load estimation for incentive based demand response [46], and also can help to enhance the frequency stability of microgrid with high penetration of renewable energy sources [47].

\section{REFERENCES}

1] F. Wang, Z. Zhen, Z. Mi, H. Sun, S. Su, and G. Yang, "Solar irradiance feature extraction and support vector machines based weather status pattern recognition model for short-term photovoltaic power forecasting," Energy Build., vol. 86, pp. 427-438, Jan. 2015, DOI: 10.1016/j.enbuild.2014.10.002

[2] F. Wang, Z. Zhen, B. Wang and Z. Mi, "Comparative Study on KNN and SVM Based Weather Classification Models for Day Ahead Short-Term Solar PV Power Forecasting," Appl. Sci., vol. 8, no. 1, pp. 28, Dec, 2017, DOI: 10.3390/app8010028

[3] M. Rana, I. Koprinska, and V. G. Agelidis, "Univariate and multivariate methods for very short-term solar photovoltaic power forecasting," Energy Convers. Manag., vol. 121, pp. 380-390, May. 2016, DOI: 10.1016/j.enconman.2016.05.025

[4] Y. Sun, F. Wang, B. Wang, Q. Chen, N.A. Engerer and Z. Mi, "Correlation feature selection and mutual information theory based quantitative research on meteorological impact factors of module temperature for solar photovoltaic systems," Energies, vol. 10, no. 1, pp. 7, Dec. 2016, DOI: 10.3390/en10010007

[5] Q. Chen, F. Wang, B.M. Hodge, J. Zhang, Z. Li, M. Shafie-khah and J.P.S. Catalão, "Dynamic Price Vector Formation Model Based Automatic Demand Response Strategy for PV-assisted EV Charging Station," IEEE Trans. Smart Grid, vol. 8, no. 6, pp. 2903-2915, Nov. 2017, DOI: 10.1109/TSG.2017.2693121

[6] F. Wang, L. Zhou, H. Ren, X. Liu, S. Talari, M. Shafie-khah and J. P.S. Catalão, "Multi-Objective Optimization Model of Source-Load-Storage Synergetic Dispatch for a Building Energy Management System Based on TOU Price Demand Response," IEEE Trans. Industry Applications, vol. 54, no. 2, pp. 1017-1028, Mar. 2018, DOI: 10.1109/TIA.2017.2781639

[7] F. Wang, Z. Zhen, C. Liu, Z. Mi, B. Hodge, M. Shafie-khah, and J. P. S. Catalão, "Image phase shift invariance based cloud motion displacement vector calculation method for ultra-short-term solar PV power forecasting," Energy Convers. Manag., vol. 157, pp. 123-135, Jan. 2018, DOI: 10.1016/j.enconman.2017.11.080

[8] F. Wang, Z. Mi, S. Su, and H. Zhao, "Short-Term Solar Irradiance Forecasting Model Based on Artificial Neural Network Using Statistical Feature Parameters," Energies, vol. 5, pp. 1355-1370, May. 2012, DOI: 10.3390/en5051355

[9] F. Wang, H. Xu, T. Xu, K. Li, M. Shafie-khah, and J. P.S. Catalão, "The values of market-based demand response on improving power system reliability under extreme circumstances," Appl. Energy, vol. 193, pp. 220-231, May. 2017, DOI: 10.1016/j.apenergy.2017.01.103 
[10] O. Noureldeen and I. Hamdan, "Design of robust intelligent protection technique for large-scale grid-connected wind farm," Prot. Control Mod. Power Syst, vol. 3, no. 3, pp. 169-182, Apr. 2018, DOI 10.1186/s41601-018-0090-4

[11] J. Li, S. Wang, L. Ye and J. Fang, "A coordinated dispatch method with pumped-storage and battery-storage for compensating the variation of wind power," Prot. Control Mod. Power Syst, vol. 3, no. 3, pp. 21-34, Jun. 2018 , DOI 10.1186/s41601-017-0074-9

[12] Shi J, Lee WJ, Liu Y, Yang Y, and Wang P, "Forecasting power output of photovoltaic systems based on weather classification and support vector machines," IEEE Trans. Ind. Appl., vol. 48, pp. 1064-1069, Feb. 2012, DOI: 10.1109/TIA.2012.2190816.

[13] Mellit A, Massi Pavan A, and Lughi V, "Short-term forecasting of power production in a large-scale photovoltaic plant," Sol Energy, vol. 105, pp. 401-413, Mar. 2014, DOI: 10.1016/j.solener.2014.03.018.

[14] Almeida MP, Perpiñán O, and Narvarte L, "PV power forecast using a nonparametric PV model," Sol Energy, vol. 115, pp 354-368, Mar. 2015, DOI: 10.1016/j.solener.2015.03.006.

[15] Z. Wang, F. Wang, and S. Su, "Solar irradiance short-term prediction model based on BP neu-ral network," Energy Procedia, vol. 12, pp. 488-494, Oct. 2011, DOI: 10.1016/j.egypro.2011.10.065

[16] Y. Sun, F. Wang, Z. Zhen, Z. Mi, C. Liu, B Wang, and J. Lu, "Research on short-term module temperature prediction model based on BP neural network for photovoltaic power forecasting," IEEE Power \& Energy Society General Meeting, pp. 1-5, July. 26-30, 2015, Denver, CO, USA, DOI: 10.1109/PESGM.2015.7286350

[17] D. Prasad Mukherjee and S. T. Acton, "Cloud tracking by scale space classification," Geosci. Remote Sensing, IEEE Trans., vol. 40, pp. 405-415, Mar. 2002, DOI: $10.1109 / 36.992803$

[18] R. Stuhlmann, M. Rieland, and E. Raschke, “An Improvement of the IGMK Model to Derive Total and Diffuse Solar Radiation at the Surface from Satellite Data," J. Appl. Meteorol., vol. 29, pp. 586-603, Jul. 1990, DOI: 10.1175/15200450(1990)029<0586:AIOTIM>2.0.CO;2

[19] H. Escrig et al., "Cloud detection, classification and motion estimation using geostationary satellite imagery for cloud cover forecast," Energy, vol. 55, no. Supplement C, pp. 853-859, Jan. 2013, DOI: 10.1016/j.energy.2013.01.054

[20] Z. Peng, S. Yoo, D. Yu, and D. Huang, "Solar irradiance forecast system based on geostationary satellite," in 2013 IEEE Conference Smart Grid Communications, pp. 708-713, Oct. 21-24, 2013, Vancouver, BC, Canada, DOI 10.1109/SmartGridComm.2013.6688042

[21] Z. Peng, D. Yu, D. Huang, J. Heiser, and P. Kalb, “A hybrid approach to estimate the complex motions of clouds in sky images," Sol. Energy, vol. 138, no. Supplement C, pp. 10-25, Sept. 2016, DOI: 10.1016/j.solener.2016.09.002

[22] C. W. Chow et al., "Intra-hour forecasting with a total sky imager at the UC San Diego solar energy testbed," Sol. Energy, vol. 85, no. 11, pp. 2881-2893, Aug. 2011, DOI: 10.1016/j.solener.2011.08.025

[23] H. Huang et al., "Cloud motion estimation for short term solar irradiation prediction," in 2013 IEEE Conference Smart Grid Communications, pp. 696-701, Oct. 21-24, 2013, Vancouver, BC, Canada, DOI: 10.1109/SmartGridComm.2013.6688040

[24] Z. Peng, D. Yu, D. Huang, J. Heiser, S. Yoo, and P. Kalb, “3D cloud detection and tracking system for solar forecast using multiple sky imagers," Sol. Energy, vol. 118, pp. 496-519, Aug. 2015, DOI: 10.1145/2554850.2554913

[25] B. K. P. Horn and B. G. Schunck, "Determining optical flow," Artif. Intell., vol. 17, no. 1, pp. 185-203, Feb. 1981, DOI: 10.1016/0004-3702(81)90024-2

[26] B. D. Lucas and T. Kanade, "An iterative technique of image registration and its application to stereo," Artif. Intell., vol. 9, no. 7, pp. 78-89, Oct. 1981, DOI: 10.1109/HPDC. 2004.1323531

[27] P. Wood-Bradley, J. Zapata, and J. Pye, "Cloud tracking with optical flow for short-tem solar forecasting," in 2012 IEEE International Conference on the 50th Conference of the Australian Solar Energy Society, pp. 55-57, Mar. 15-18, 2012, Mawson, SA, AUS, DOI: 10.1016/j.solener.2015.03.030

[28] C. W. Chow, S. Belongie, and J. Kleissl, "Cloud motion and stability estimation for intra-hour solar forecasting," Sol. Energy, vol. 115, no. Supplement C, pp. 645-655, Mar. 2015, DOI: 10.1016/j.solener.2015.03.030

[29] T. Brox and J. Malik, "Large Displacement Optical Flow: Descriptor Matching in Variational Motion Estimation," IEEE Trans. Pattern Anal. Mach. Intell., vol. 33, pp. 500-513, Mar. 2011, DOI: 10.1109/TPAMI.2010.143

[30] H.-Y. Cheng and C.-C. Yu, "Solar irradiance now-casting with ramp-down event prediction via enhanced cloud detection and tracking," in 2016 IEEE International Conference on Multimedia and Expo, pp. 11-17, July. 11-15, 2016 , Seattle, WA, USA, DOI: 10.1109/ICME.2016.7552863
[31] F. Su, W. Jiang, J. Zhang, H. Wang, and M. Zhang, "A local features-based approach to all-sky image prediction," IBM J. Res. Dev., vol. 59, pp. 6:1-6:10, May 2015, DOI: 10.1147/JRD.2015.2397772

32] Xian GM, "An identification method of malignant and benign liver tumors from ultrasonography based on GLCM texture features and fuzzy SVM," Expert Syst Appl., vol. 37, pp. 6737-6741, Feb. 2010, DOI 10.1016/j.eswa.2010.02.067.

[33] S. Su et al., "A classified irradiance forecast approach for solar PV prediction based on wavelet decomposition," in 2016 International Conference on North American Power Symposium, pp. 1-5, Sept. 18-20, 2016, Waterfront, MGW, USA, DOI: 10.1109/NAPS.2016.7747957

[34] Hamill TM, Nehrkorn T. "A Short-Term Cloud Forecast Scheme Using Cross Correlations," Weather Forecast, vol. 8, pp. 401-411, Aug. 1993, DOI: 10.1175/1520-0434(1993)008<0401:ASTCFS >2.0.CO;2.

[35] C. Willert and M. Gharib, "Digital Particle Image Velocimetry," Exp. Fluids, vol. 10, pp. 181-193, Jan. 1991, DOI: 10.1007/BF00190388

[36] B. Yu, L. Wang, and Z. Niu, "A novel algorithm in buildings/shadow detection based on Harris detector," Opt. - Int. J. Light Electron Opt., vol. 125, no. 2, pp. 741-744, July. 2014, DOI: 10.1016/j.ijleo.2013.07.043

[37] W.-L. Zhao and C.-W. Ngo, "Flip-Invariant SIFT for Copy and Object Detection," IEEE Trans. Image Process, vol. 22, no. 3, pp. 980-991, Oct. 2012, DOI 10.1109/TIP.2012.2226043

[38] F. Wang, L. Zhou, H. Ren and X. Liu, "Search Improvement Process-Chaotic Optimization-Particle Swarm Optimization-Elite Retention Strategy and Improved Combined Cooling-Heating-Power Strategy Based Two-Time Scale Multi-Objective Optimization Model for Stand-Alone Microgrid Operation," Energies, vol. 10, no. 12, pp.1936-1942, Dec. 2017, DOI: 10.3390/en10121936

[39] F. Wang, L. Zhou, B. Wang, Z. Wang, M. Shafie-khah, and J. P. S. Catalão, "Modified Chaos Particle Swarm Optimization-Based Optimized Operation Model for Stand-Alone CCHP Microgrid," Appl. Sci., vol. 7, no. 8, pp. 754-759, Jul. 2017, DOI: 10.3390/app7080754

[40] Z. Zhen, F. Wang, Y. Sun, Z. Mi, C. Liu, B. Wang and J. Lu, "SVM based cloud classification model using total sky images for PV power forecasting," in Proc. IEEE Power and Energy Society Innovative Smart Grid Technologies Conference (ISGT), pp. 18-20, Feb. 18-20, 2015, Washington, DC, USA, DOI: 10.1109/ISGT.2015.7131784

[41] F. Wang, K. Li, L. Zhou, H. Ren, J. Contreras, M. Shafie-khah and J.P.S. Catalão, "Daily pattern prediction based classification modeling approach for dayahead electricity price forecasting," Int. J. Electr. Power Energy Syst., vol. 105, pp. 529-540, Feb. 2019. https://doi.org/10.1016/j.ijepes.2018.08.039.

[42] F. Wang, Z. Zhang, C. Liu, Y. Yu, S. Pang, N. Duić, M. Shafie-khah and J. P. S Catalão, "Generative adversarial networks and convolutional neural networks based weather classification model for day ahead short-term photovoltaic power forecasting," Energy Convers. Manag., vol. 181, pp. 443-462, Feb. 2019, DOI 10.1016/j.enconman.2018.11.074

[43] F. Wang, Z. Mi, S. Su, C. Zhang, "A practical model for single-step power prediction of grid-connected PV plant using artificial neural network," 2011 IEEE PES Innovative Smart Grid Technologies (ISGT), pp. 1-4, Nov. 13-16, 2011, Perth, WA, AUS, DOI: 10.1109/ISGT-Asia.2011.6167097.

[44] F. Wang, S. Pang, Z. Zhen, K. Li, H. Ren, M. Shafie-khah, and J. P. S. Catalão, "Pattern Classification and PSO Optimal Weights Based Sky Images Cloud Motion Speed Calculation Method for Solar PV Power Forecasting," in Proc. IEEE Ind. Appl. Soc. Annu. Meeting (IEEE IAS AM), pp. 1-9, Sep. 23-27, 2018 Portland, OR, USA, DOI: 10.1109/IAS.2018.8544468

[45] F. Wang, K. Li, N. Dui, Z. Mi, B.M. Hodge, M. Shafie-khah and J.P.S. Catalao, "Association rule mining based quantitative analysis approach of household characteristics impacts on residential electricity consumption patterns," Energy Convers. Manag., vol. 171, pp. 839-854, Sep. 2018, DOI: 10.1016/j.enconman.2018.06.017

[46] F. Wang, K. Li, C. Liu, Z. Mi, M. Shafie-khah and J.P.S. Catalão, "Synchronous Pattern Matching Principle Based Residential Demand Response Baseline Estimation: Mechanism Analysis and Approach Description," IEEE Trans. Smart Grid, vol. 9, no. 6, pp. 6972-6985, Apr. 2018, DOI: 10.1109/TSG.2018.2824842

[47] X. Zhu, M. Xia, H. D. Chiang, "Coordinated sectional droop charging control for EV aggregator enhancing frequency stability of microgrid with high penetration of renewable energy sources," Appl. Energy, vol. 210, pp. 936-943, Jan. 2018. DOI: 10.1016/j.apenergy.2017.07.087 


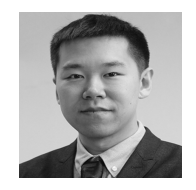

Zhao Zhen received the B.S. degree and Ph.D. degrees in electrical engineering from North China Electric Power University (NCEPU), Baoding, China, in 2012, 2018, respectively. Currently, he is an assistant professor with the Department of Electrical Engineering at NCEPU, Baoding, China. His research interests include solar irradiance and power forecasting.

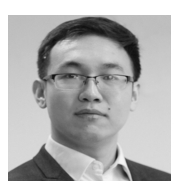

Shuaijie Pang received the B.S. degree in electrical engineering from North China Electric Power University (NCEPU), Baoding, China, in 2016. Currently, he is a master student with the Department of Electrical Engineering at NCEPU, Baoding, China. His research interests include solar irradiance forecasting and machine learning.

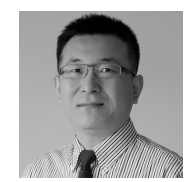

Fei Wang (M'09-SM'17) received the B.S. degree from Hebei University, Baoding, China in 1993, the M.S. and Ph.D. degrees in electrical engineering from North China Electric Power University (NCEPU), Baoding, China, in 2005 and 2013, respectively. Currently, he is a Professor with the Department of Electrical Engineering and the State Key Laboratory of Alternate Electrical Power System with Renewable Energy Sources at NCEPU, Baoding and Beijing, China. He is the Director of Smart Energy Network Integrated Operation Research (SENIOR) Center at NCEPU. He was a visiting Professor with the Department of Electrical and Computer Engineering, University of Illinois at Urbana-Champaign, Urbana, IL, USA, from 2016 to 2017. He was a researcher with the Department of Electrical Engineering at Tsinghua University, Beijing, China, from 2014 to 2016.

Prof. Wang is an Editor of IET Renewable Power Generation and the expert member of IEC SC8A/WG2. He was the recipient of the 2018 Technical Invention First Award of Hebei Province, the 2018 Patent Award of Hebei Province, the 2014 Natural Sciences Academic Innovation Achievement Award of Hebei Province, the 2018 China Electric Power Science and Technology Progress Award, and the 2014 Outstanding Doctoral Dissertation Award of NCEPU. He was the General Chair of the 2017 International Seminar of Renewable Energy Power Forecasting and Absorption Technology and 2018 International Seminar of Integrated Energy and Smart Microgrid Technology. He was the Guest Editor for the Special Issue on "Demand Side Management and Market Design for Renewable Energy Support and Integration" of IET Renewable Power Generation. His research interests include renewable energy power, electricity price and load forecasting; demand response and electricity market; smart grid; microgrid; and integrated energy system.

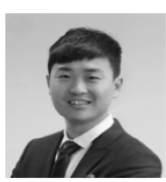

Kangping Li (SM'17) received the B.S., M.S. and Ph.D. degrees degree in electrical engineering from North China Electric Power University (NCEPU), Baoding, China, in 2015. Currently he is a Ph.D. student with the Department of Electrical Engineering at NCEPU, Baoding, China. His research interests include electricity market and demand response.

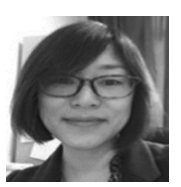

Hui Ren received the M.S. and Ph.D. degree in electrical engineering from North China Electric Power University (NCEPU), Baoding, China, in 1997, 2009, respectively. She is currently a Professor with the Department of Electrical and Electronic Engineering, NCEPU. She was a Visiting Professor at the University of Wisconsin-Madison, Madison, WI, USA, from 2006 to 2007, and at the Pontificia Universidad Cato lica de Chile, from 2014 to 2015 and July 2016 to October 2016. Her research interests include the planning and operation of active distribution network, risk analysis and early warning of large-scale blackouts.

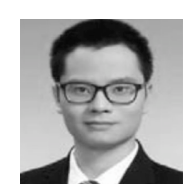

Zhigang Li (S'13, M'16) received the B.S and Ph.D. degrees in electrical engineering from Tsinghua University, Beijing, China, in 2011 and 2016, respectively. He is currently an Associate Professor with the School of Electric Power, South China University of Technology, Guangzhou, China. He was a
Visiting Scholar at the Illinois Institute of Technology, Chicago, IL, USA, and the Argonne National Laboratory, Argonne, IL, USA, from 2014 to 2015. His research interests include renewable energy integration, power system optimization, and integrated energy systems.

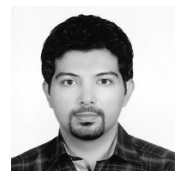

Miadreza Shafie-khah (M'13, SM'17) received the M.Sc and Ph.D. degrees in electrical engineering from Tarbiat Modares University, Tehran, Iran, in 2008 and 2012, respectively. $\mathrm{He}$ received his first postdoc from the University of Beira Interior (UBI), Covilha, Portugal in 2015. He received his second postdoc from the University of Salerno, Salerno, Italy in 2016. Currently, he is an Assistant Professor at the University of Vaasa, Vaasa, Finland. He was considered one of the Outstanding Reviewers of the IEEE TRANSACTIONS ON SUSTAINABLE ENERGY, in 2014 and 2017, one of the Best Reviewers of the IEEE TRANSACTIONS ON SMART GRID, in 2016 and 2017, and one of the Outstanding Reviewers of the IEEE TRANSACTIONS ON POWER SYSTEMS, in 2017 and 2018. His research interests include power market simulation, market power monitoring, power system optimization, demand response, electric vehicles, price forecasting and smart grids.

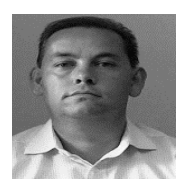

João P. S. Catalão (M'04-SM'12) received the M.Sc. degree from the Instituto Superior Técnico (IST), Lisbon, Portugal, in 2003, and the Ph.D. degree and Habilitation for Full Professor ("Agregação") from the University of Beira Interior (UBI), Covilha, Portugal, in 2007 and 2013, respectively. Currently, he is a Professor at the Faculty of Engineering of the University of Porto (FEUP), Porto, Portugal, and Research Coordinator at INESC TEC. $\mathrm{He}$ was also appointed as Visiting Professor by North China Electric Power University, Beijing, China. He was the Primary Coordinator of the EU-funded FP7 project SiNGULAR ("Smart and Sustainable Insular Electricity Grids Under Large-Scale Renewable Integration"), a 5.2-million-euro project involving 11 industry partners. He has authored or coauthored more than 700 publications, including 275 journal papers (more than 80 IEEE Transactions/Journal papers), 372 conference proceedings papers, 5 books, 35 book chapters, and 14 technical reports, with an $h$-index of 47 , an $i 10$-index of 197 , and over 8500 citations (according to Google Scholar), having supervised more than 70 post-docs, Ph.D. and M.Sc. students. He is the Editor of the books entitled "Electric Power Systems: Advanced Forecasting Techniques and Optimal Generation Scheduling'" and "Smart and Sustainable Power Systems: Operations, Planning and Economics of Insular Electricity Grids" (Boca Raton, FL, USA: CRC Press, 2012 and 2015 , respectively). His research interests include power system operations and planning, hydro and thermal scheduling, wind and price forecasting, distributed renewable generation, demand response and smart grids.

Prof. Catalão is an Editor of the IEEE Transactions on Smart Grid, an Editor of the IEEE Transactions on Power Systems, an Associate Editor of the IEEE Transactions on Industrial Informatics. From 2011 till 2018 (seven years) he was an Editor of the IEEE Transactions on Sustainable Energy and an Associate Editor of the IET Renewable Power Generation. He was also a Subject Editor of the IET Renewable Power Generation. He was the Guest Editor-in-Chief for the Special Section on "Real-Time Demand Response" of the IEEE Transactions on Smart Grid, published in December 2012, the Guest Editor-in-Chief for the Special Section on "Reserve and Flexibility for Handling Variability and Uncertainty of Renewable Generation" of the IEEE Transactions on Sustainable Energy, published in April 2016, the Corresponding Guest Editor for the Special Section on "Industrial and Commercial Demand Response" of the IEEE Transactions on Industrial Informatics, published in November 2018, and the Lead Guest Editor for the Special Issue on "Demand Side Management and Market Design for Renewable Energy Support and Integration" of the IET Renewable Power Generation, to be published in March 2019. He was the recipient of the 2011 Scientific Merit Award UBI-FE/Santander Universities, the 2012 Scientific Award UTL/Santander Totta, the 2016 and 2017 FEUP Diplomas of Scientific Recognition, the 2017 Best INESC-ID Researcher Award, and the 2018 Scientific Award ULisboa/Santander Universities, in addition to an Honorable Mention in the 2017 Scientific Award ULisboa/Santander Universities. Moreover, he has won 4 Best Paper Awards at IEEE Conferences. 